\title{
Review of: "Identifying patients with symptoms suspicious for COVID-19 at elevated risk of adverse events: The COVAS score"
}

\author{
Xavier Roux ${ }^{1}$ \\ 1 Geneva University Hospital
}

Potential competing interests: The author(s) declared that no potential competing interests exist.

Interesting paper about a score of prediction of complication of covid 19 disease. A low score seems intéressant to rule out a bad outcome and allow a discharge from ED. The main limitation is about population selection with a lack of Sarscov2 test in many of them. For patients with clinical suspicion of pneumonia, the AUC is not so different comparatively to the others score (CURB65). The likelihood ratio and the youden index could be put on results and dicussion. 